\title{
Test and Analysis of Short Electric Arc Trepanning Machining of Difficult-to-cut Material
}

\author{
Hai Peng ${ }^{1, a}$ and Zhaoyuan Zhang ${ }^{2, b}$ \\ ${ }^{1,2}$ School of Mechanical Engineering, Xi'an Shiyou University, Xi'an, Shannxi (710065), China \\ ahpeng1104@sina.com, b1044131624@qq.com
}

Keyword: Short electric arc; Difficult-to-cut materials; Trepanning machining

\begin{abstract}
Short electric arc machining is a new type of special processing technology, and the method has many advantages such as no cutting force, high processing efficiency and little influence on thermal deformation, etc. In this paper, the features of several typical difficult-to-cut materials were introduced. And the principle and mechanism of short electric arc machining were analyzed. According to the trepanning machining characteristic, the system of the short electric arc in trepanning machining and the electrode tip were designed. By analyzing and comparing the experimental data, the relationship between the discharge area and peak current size was identified, the relationship between covering effect on the depth of material and electrode loss was identified, the relationship between different workpiece materials and processing speed was identified. The results of analysis and test show that the method of short electric arc trepanning processing has the feature of no cutting force, tool electrode is easy to manufacture, machining efficiency and is easy to control etc., it is an exploration of new methods to the difficult-to-cut material trepanning machining.
\end{abstract}

\section{Introduction}

Rapid scientific technology development and progress of industrial production, an increasing number of new materials with high hardness, high strength, high temperature resistance, good wear resistance and corrosion resistance have been used in aerospace, military industry and oil fields. These new materials will lead to high cutting force, high cutting temperature and cutting tool wears in the machining process, which will cause difficulties using traditional mechanical machining methods, especially for difficult-to-cut material trepanning machining difficulty bigger. For these reasons, non-traditional machining method is considered to be one of the effective ways to solve this problem. Short electric arc machining technique is characterized by no cutting force, tool electrode characteristics of easy manufacturing, machining of high efficiency and easy to control. Therefore short electric arc machining technique has some achievements in the field of super hard and super difficult to machine materials and this technology is considered to be a good a kind of good prospects for development of special processing technology, which provides a new research direction for deep hole trepanning machining.

\section{The Processing Characteristics of Typical Difficult-To-Cut Materials}

Difficult-to-cut materials has the feature of high strength, high hardness, high wear resistance and corrosion resistance etc, so there exists high cutting force, cutting temperature and tool wear fast machining characteristics. Typical difficult-to-cut materials include Stainless steel, Titanium alloy, Ni-base high-temperature alloy, etc.

The Processing Characteristics of Stainless Steel Material. Precipitation hardening stainless steel $(0 \mathrm{Cr} 17 \mathrm{Ni} 4 \mathrm{Cu} 4 \mathrm{Nb})$ with high strength $\left(\sigma_{b} \geq 1100 \mathrm{MPa}\right)$, hardness (up to HRC 40$)$ and corrosion resistance. Its machinability is poor, prone to work hardening in the machining process. Due to the high hot strength of material, the processing will produce larger cutting force and high cutting temperature, and the effect will makes the tool and chip bonding and diffusion. The chip is not easy to break but easy to adhesion, which the rake face on tool will friction serious, easy to form a built-up edge under high temperature and high pressure, increases tool wear, damage machining 
surface quality.

The Processing Characteristics of Titanium Alloy Material. Titanium alloy with lower density, higher specific strength, high temperature resistant, corrosion resistance and other excellent physical and mechanical properties, are widely used in aviation, aerospace and other defense area. Titanium alloy materials have high thermal strength, small thermal conductivity, a hardened layer and a brittle layer can be formed at $600{ }^{\circ} \mathrm{C}$, causing high cutting force and cutting temperature. At the same time, the elastic modulus of titanium alloy is low, likely to produce elastic deformation, and caused a large amount of spring back in the machining surface and make the rear face of the cutting tool has adhesion wear [1].

The Processing Characteristics of High-temperature Alloy Material. High-temperature alloy has excellent high temperature strength, thermal stability and thermal fatigue resistance. The content of nickel in nickel base super alloy reach more than $45 \%$, and it has excellent high temperature oxidation resistance.

High temperature alloy is one of the most difficult to machining in all kinds of difficult-to-cut materials, the softening temperature is higher, softening rate is low, consequently, lead the hardening phenomenon seriously. The high temperature strength of high temperature alloys is very high, deformation resistance of the process is rapid rise and the cold work hardening is serious, which enlarge the cutting force. The cutting temperature is extremely high, even reach $1000{ }^{\circ} \mathrm{C}$ when machining high temperature alloys, this temperature arouse tool vulnerable to deform and wear. Cutting could bring about adhesion, diffusion, oxidation, groove wear and the tool life degree rapidly, processing precision hardly insure [2].

\section{Principle and Mechanism Analysis of Short Electric Arc Machining}

Short electric arc machining is a new type of special processing technology, which uses the high temperature of arc discharge between the workpiece and tool electrode to corrosion the excess metal, and the material which can be removed from the workpiece by the mixture of certain pressure gas-liquid, and to achieve the required size of the workpiece [3]. The working power supply is a kind of low voltage large current $(0 \sim 30 \mathrm{~V}, 0 \sim 3000 \mathrm{~A})$ of the pulse power supply, its working principle as shown in Fig.1. The machining workpiece is installed on the machine tool spindle to do a rotary motion, connected with the positive electrode. The tool electrode is connected with the negative electrode and the relative motion of the main shaft is driven by the motor [4]. In the process of machining, with a certain pressure gas-liquid mixing medium injected into the processing zone. When the gap of the workpiece material and the tool electrode is less than the critical distance of arc discharge, cutting area will produce intense discharge phenomena and the high temperature that discharge produce will melt surface of workpiece material partial, under the impact of the medium, the melted material is detached from the machined surface. Thus for the high efficiency machining of the workpiece without cutting force [5].

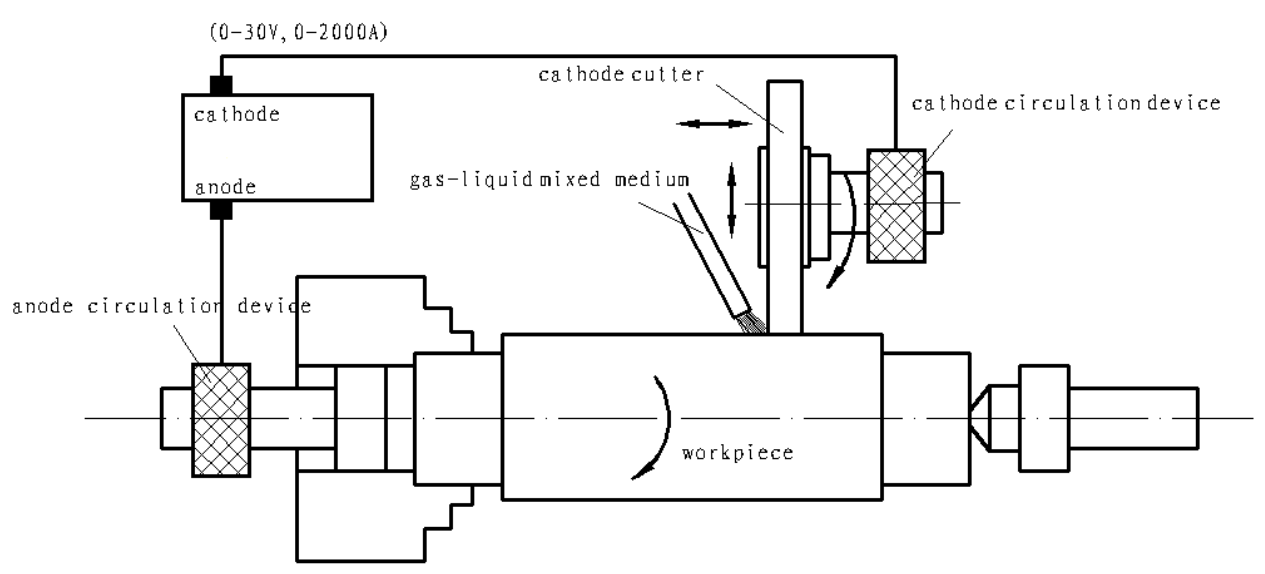

Figure 1. Schematic diagram of short electric arc machining 
In the short electric arc machining, when the tool cutter and the workpiece close to each other, electric field strength between the electrodes is gradually increased to the current to breakdown the working medium. With arc discharge the cathode surface escape electron under the electric field force and then high speed to the anode movement producing a large number of heat, so that the workpiece surface temperature increased sharply. The heat will melt metal materials even boiling gasification, the gasification of the steam could instantaneous volume soared and expanded and explosive. Just melted and not stripped molten metal droplets are condensing on the machined surface by the cooling working medium. On this account complete the process of electrical corrosion [6].

\section{Test and Analysis of Short Electric Arc Trepanning Machining}

The Short Electric Arc Trepanning Machining System. According to the principle of short electric arc machining and the requirements of trepanning machining, trepanning machining system of short electric arc was designed. Fig. 2 for processing system schematic diagram. The electric arc power supply is DHX33A3000/28FS short arc cutting power supply, the working current can reach $2000 \mathrm{~A}$, the voltage range of $0 \sim 30 \mathrm{~V}$, the power operation panel is shown in Fig. 3. The rear end of the lathe spindle is provided with an anode circulation device to connect the workpiece to the positive electrode and the drill pipe is connected with the cathode circulation device to make the drill pipe connected with the negative electrode and take measures to make the machine tool insulation. Working medium with a certain pressure of water vapor mixture of emulsified cutting fluid medium and the test of the workpiece material for precipitation hardening stainless steel $(0 \mathrm{Cr} 17 \mathrm{Ni} 4 \mathrm{Cu} 4 \mathrm{Nb})$ and titanium alloy (TC4).

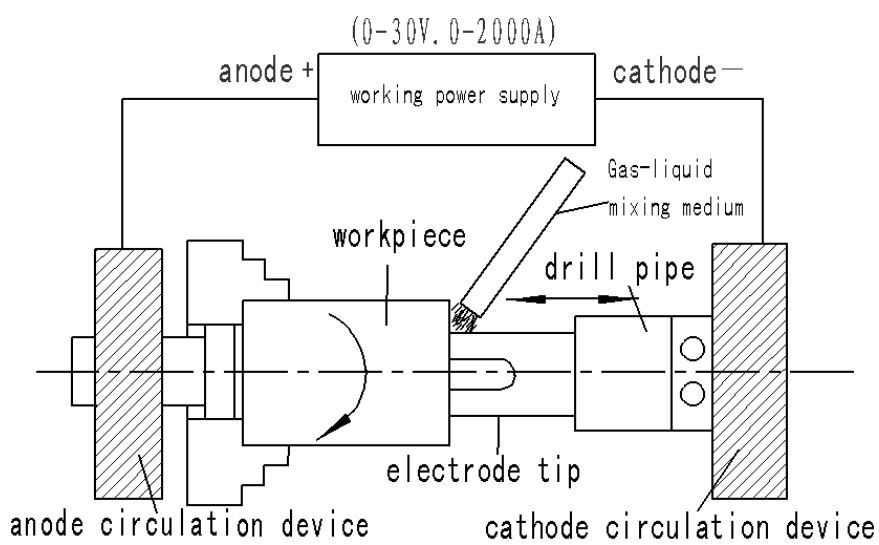

Figure 2. The short electric arc trepanning machining

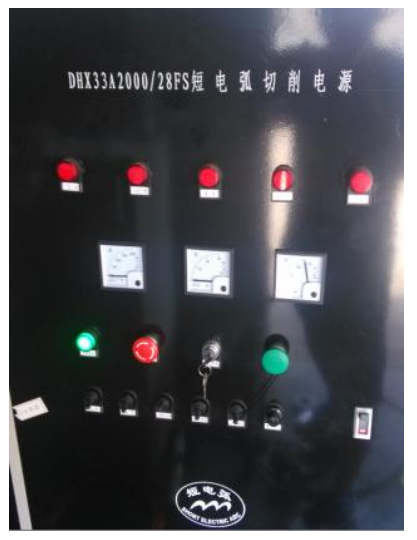

Figure.3 The short electric arc cutting power

When machining the processed workpiece and the electrode tip in the drive system of the drive of each other to do the rotating movement, at the beginning, the machine automatic feeding system has a certain distance between the workpiece and the electrode tip, so as to form a discharge gap. At this moment the certain pressure of the water vapour mixture will into the working area, when the distance of workpiece and electrode tip to discharge gap began intense discharge, the high temperature of the arc generated by the workpiece surface discharge area will make material rapidly melting and gasification then the material to be removed by the work medium cooling, stripping and chip removal, and forming the new discharge process, thus accomplishes the workpiece trepanning machining.

The Design and Material Selection of Electrode Tip. The traditional deep hole trepanning processing is adopted deep hole trepanning drill, for the short electric arc trepanning processing should be designed to meet the requirements of electric arc machining drill, this is the electrode tip. The electrode tip is mainly reflected in the electric arc discharge between the tool electrode (electrode tip) and workpiece, to complete the removal in metal materials, in order to ensure the machining accuracy the electrode tip to play a guiding role in the machining process. The electrode 
tip material will affect the processing characteristics and efficiency, therefore, the corresponding experiments were using two kinds of materials (ductile iron and 45 steel), and the size and shape of the electrode tip are shown in Fig. 4 and Fig. 5. When machining, the electrode tip and the workpiece are processed in the same center line, four tooth profile heads at the front of the electrode tip are gradually close to the end face of the workpiece. When discharge gap reached the arc discharge is generated, thus for to carry out short electric arc discharge trepanning machining.
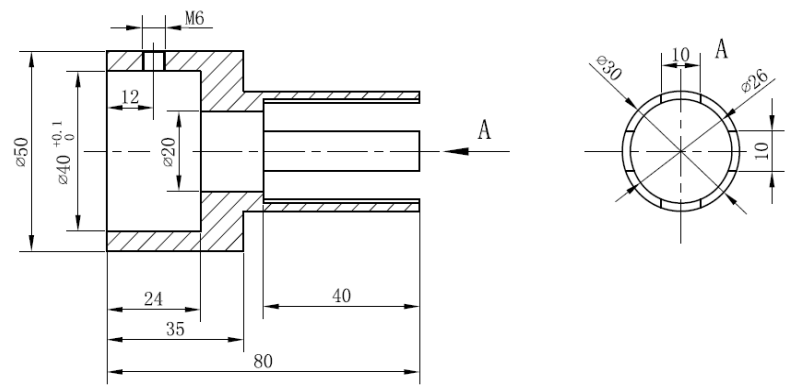

Figure 4. $\Phi 30 \mathrm{~mm}$ electrode tip
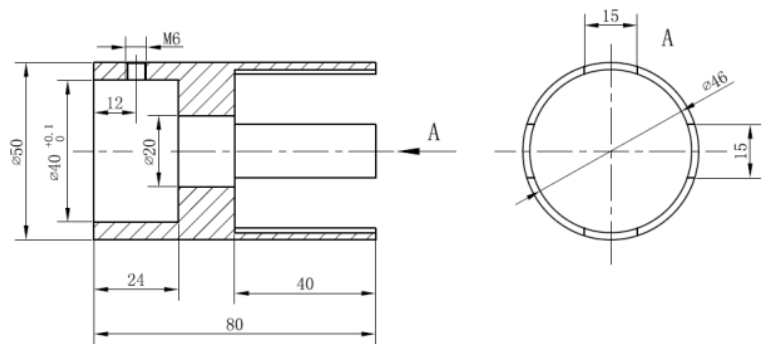

Figure 5. $\Phi 50 \mathrm{~mm}$ electrode tip

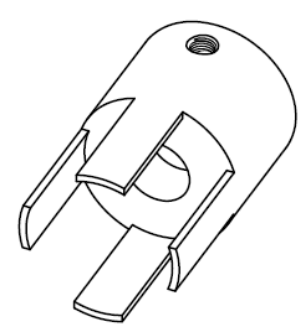

Test and Analysis of Electric Arc Trepanning Machining. In this experiment, two kinds of electrode tip were used in the electric arc trepanning machining, the material of one electrode tip is 45 steel, the machining diameter is $30 \mathrm{~mm}$, respectively processing the outside diameter of $50 \mathrm{~mm}$ titanium alloy and outer diameter of $70 \mathrm{~mm}$ stainless steel. Another kind of electrode tip materials is ductile iron, the machining diameter is $50 \mathrm{~mm}$, processing the outside diameter of $70 \mathrm{~mm}$ stainless steel.

After installation the workpiece and electrode tip, set the different power supply parameters (change the size of voltage) and the corresponding feed. When machining the workpiece rotation the electrode tip automatically feed and washing by high-pressure gas-liquid mixing medium, so as to get processed workpiece the processing is shown in Fig. 6.

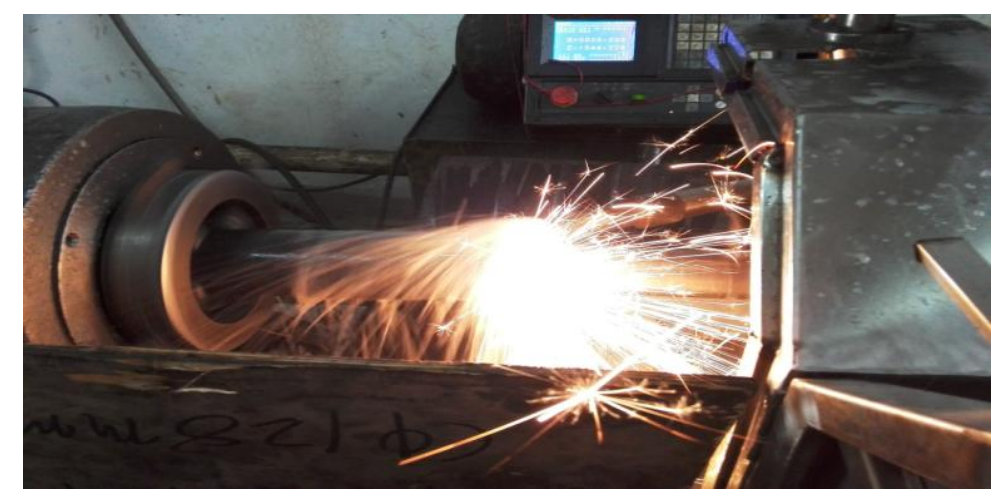

Figure 6. Short electric arc deep hole trepanning machining

After completion of processing then observe the processing effect of the workpiece and the electrode tip loss and to measure processed depth and machining quality of the workpiece and the 
wear amount of electrode tip. Then replace workpiece and electrode tips and further the processing test. Fig. 7 shows the machining diameter of $30 \mathrm{~mm}$ electrode tip which is after processing and Fig. 8 shows the pattern of stainless steel workpiece which is after initial processing.

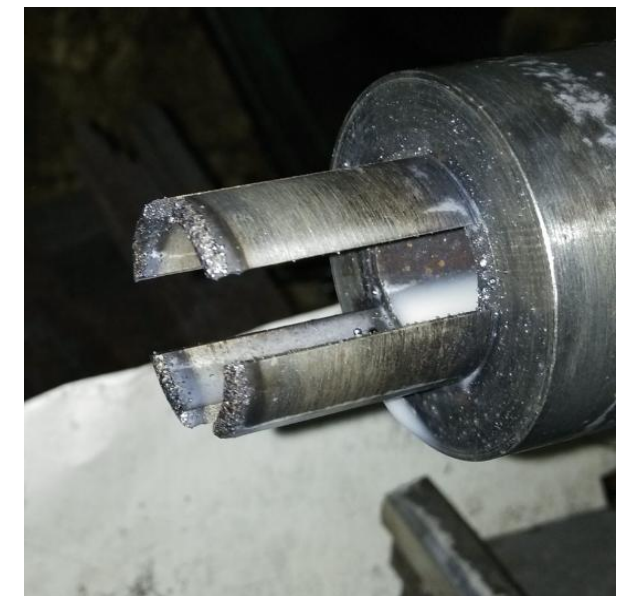

Figure 7. $\Phi 30 \mathrm{~mm}$ electrode head after processed

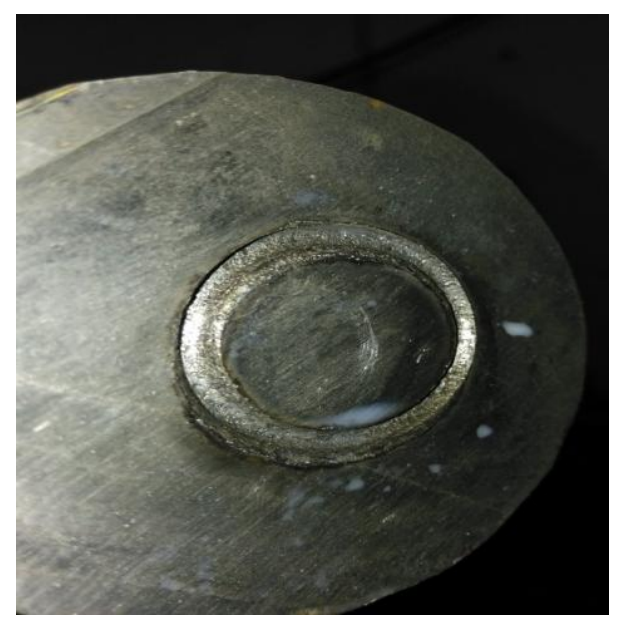

Figure 8. stainless steel after initial processed

Fig. 7 shows that the head of $\Phi 30 \mathrm{~mm}$ electrode tip which is after processing appeared arc protrusion and obvious corrosion pit. Arc protrusion is due to a small part of melting and gasification metal which is cast from electrode wills condensation on the electrode surface and then forms the covering effect. Black metal electrode tip is processed in the process of high temperature oxidation. Fig. 8 shows the pattern of stainless steel workpiece which is after initial processing. It can be seen that the end face of the stainless steel bar has a neat circular corrosion pit the width of the corrosion pit is bigger than that of the electrode tip blade and surface quality of corrosion pits groove is better.

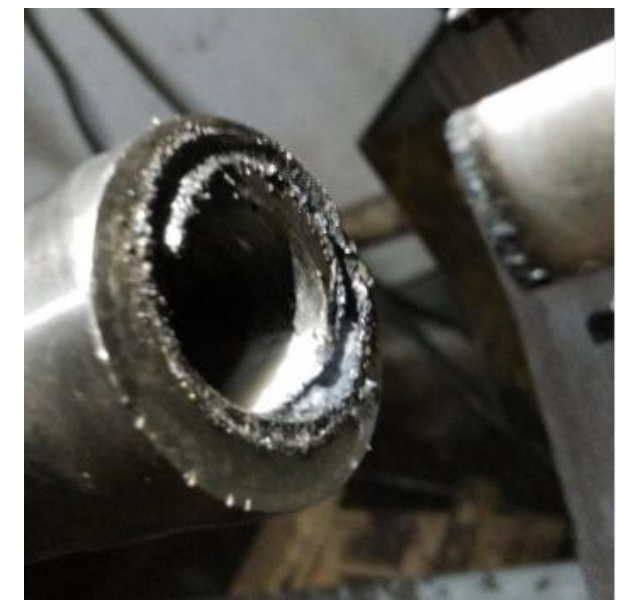

Figure 9. After processing of titanium alloy

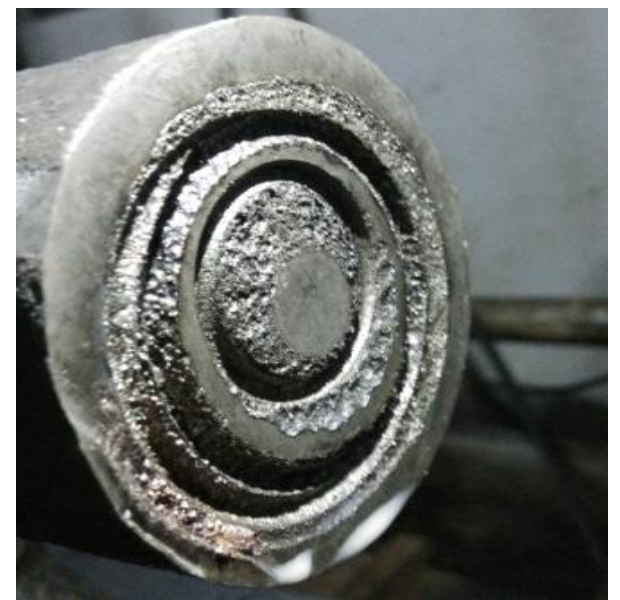

Figure 10. After processing of stainless steel

Fig. 9 and 10 respectively show the pattern of titanium alloy and stainless steel which are after repeated short arc machining. It can be seen that both two kind of materials have deep corrosion pit and the edge of corrosion pit is convex and the metallic small particles cohere on the end, but the corrosion pit of titanium alloy is significantly deeper than stainless steel. Only four toothed end face of electrode tip happen arc discharge machining with workpiece end face at the beginning of the short arc machining, along with the processing, electrode tip will gradually go into the corrosion pit. Meanwhile, the end face of four toothed and flank of the electrode tip both produce arc discharge in processing, the amount of metal removal in reach a peak state. Because of the working liquid is deficiently spraying in the region of the internal processing, the stripped high temperature metal cannot be cooling and washed in time, which will stack in the periphery of corrosion pit and form 
accumulation and particle binding [7].

Observe the wastage of the electrode by changing the voltage parameters, the electrode loss increases with the increase of feed rate when the voltage is $10 \mathrm{~V}$. On the one hand, this is the erosion increase of workpiece material, working current is increased, resulted the increase in the loss of the electrode tip material. On the other hand, with the machining depth of the electrode tip, the increase of the discharge machining area also caused the increase of electrode wear. When the voltage decreases to $6 \mathrm{~V}$, the electrode tip loss decreases, which is due to the corrosion rate of the electrode tip are proportional to the voltage and the average current. As the voltage decrease, the rate of metal removal decreases in corresponding. Another reason is the melting, gasification metal compensation effect. Another set of experiments in the feed rate of the same circumstances, with the increase of voltage, the loss of the electrode becomes smaller, and with the test of electrode loss is increasing finally. This is the gradual deepening of the electrode, the electrode tip cover effect increases, the discharge area increases, the depth and width of the material increases, the electric corrosion rate increases, the electrode loss increases, while the processing speed is also reached a peak, the processing efficiency is the highest, and then the electrode loss back to a numerical value and tends to be stable [8].

Through the analysis of two sets of experimental parameters, it can be seen that the effect of titanium alloy processing is higher than stainless steels, but the processing speed of stainless steel is generally higher than that of titanium alloy, which is due to the density of stainless steel is greater than titanium alloy, and the thermal conductivity of it is less than titanium alloy, also stainless steel get more heat, which leads to the decrease of temperature is unfavorable and to speed up the rate of metal removal. At the same time, it is observed that the loss of the electrode tip of 45 steel material processing of titanium alloy is higher than stainless steel, which is because the boiling point and melting point of the titanium alloy are higher than the 45 steel. So the electrode tip loss will be very large when processing.

When the electrode processing aperture increases processing speed the electrode will loss obviously. Because the electrode diameter and the area of the discharge machining is increased, the discharge current increases, the amount of removal metals is increased, and the electrode material with low melting point, which leads to the increase of the absolute loss of the electrode. In the processing of stainless steel material, the electrode tip loss of 45 steel is smaller than the electrode tip loss of cast iron material, which is due to the 45 steel melting boiling higher than cast iron.

Through the above test and analysis show that in the manufacturing process of short electric arc trepanning machining, the current is always rising gradually up to a peak and then drop back to a stable value. The peak current is proportional to the discharge area and the greater of the current with the electrodes loss fast. The loss of electrode increases with the voltage climb and electrode loss rule will be affected by the cover effect. The width of the trepanning increased is due to the electrode tip covering effect make the thickness of the tooth tip and the electric erosion pit width increases. In the experiment, the loss of 45 steel electrode is less than cast iron electrode. It shows that when the melting point of electrode materials get higher the loss lower. In the short electric arc machining, the properties of the material have great influence on the processing speed, when the greater of the density and lower of the thermal conductivity the material would be greater of the processing speed. The density of stainless steel is larger than titanium alloy and the thermal conductivity is lower than titanium alloy, so the metal removal rate is faster.

\section{Conclusion}

Through the theoretical analysis and related experiments, it can be concluded that short electric arc process trepanning processing is achievable, and the method can provide the corresponding basis for processing to the subsequent semi-finishing and finish-machining processing.

Through the short electric arc process trepanning experiment using several difficult-to-cut materials, it is concluded that the greater the density and the lower the thermal conductivity to materials, the faster processing speed it has. The stainless steel compared with the titanium alloy, the metal removal rate is faster in the test, so the processing speed is faster. 
Through test and analysis the design of two different kind of material and size of the electrode tip, and under the condition of different electrical parameters, it can be found that the size of peak current is related to the discharge area, the increase of the width of trepanning is associated with the covering effect of electrode tip, electrode loss is related to its material properties and covering effect. Also, the higher the melt point and boiling point of electrode material are and the smaller loss it will be too and electrode loss is inversely proportional to the voltage, is proportional to the current.

\section{References}

[1] W.H. Zhen, Y.L. Zhang and M.R. Zhan: Hard Cutting Materials Processing Technology Question and Answer (Beijing Publishing, china 2001), pp43-261, (in Chinese).

[2] H.S. Liu, J.P. Zhou and Q. Yu: Explore of the Surface Quality of Nickel-based Superalloy Based on the Short arc Machining, Vol. (2014) No.05, pp.128-130, (in Chinese).

[3] C.H. Liang, J.P. Zhou: The Short Arc Machining Technique and Its Application, Vol. (2007) No.12, pp.92-94, (in Chinese).

[4] W.Y. Lv: The Research on Short Arc Discharge Machining, Vol. (2014) No.05, pp.48-49, (in Chinese).

[5] Z.S. Wu, L.J. Yang and Z.Y. Li: Modern Electric Arc Welding Method and Equipment, (Chemical Industry Press, china 2010), pp16-20,(in Chinese).

[6] J. Lu: Study of the Mechanism on Short Arc Machining (MS. Xin Jiang University, China 2011),(in Chinese).

[7] B. Ma: The Research and Analysis of Workpiece Surface Quality By Short Arc Machining (MS. Xin Jiang University, China 2011), (in Chinese).

[8] H. He: The Research of Edm Milling Machine Tools and Key Technology Based on the Liquid Rotating Electrode (PHD, Harbin institute of technology, china 2011), (in Chinese). 